\title{
SOXI7 Antagonizes the WNT Signaling Pathway and is Epigenetically Inactivated in Clear-Cell Renal Cell Carcinoma
}

\author{
Lu Wang (iD) \\ Zhe Wang ${ }^{2}$ \\ Yuze Zhu' \\ Shutao Tan' \\ Xiaonan Chen' \\ Xianghong Yang ${ }^{2}$ \\ 'Department of Urology, Shengjing \\ Hospital of China Medical University, \\ Shenyang, I 10004, People's Republic of \\ China; ${ }^{2}$ Department of Pathology, \\ Shengjing Hospital of China Medical \\ University, Shenyang, I 10004, People's \\ Republic of China
}

Correspondence: Xianghong Yang Department of Pathology, Shengjing Hospital of China Medical University, Shenyang, I 10004, People's Republic of China

Tel +8618940251640

Email yangxh@sj-hospital.org
Background: SRY-box containing gene 17 (SOX17) was reported to be a candidate tumor suppressor gene in multiple tumors. Little is known about its role in clear-cell renal cell carcinoma (ccRCC). This study aims to identify the epigenetic regulation and tumorsuppressive function of SOX17 in ccRCC.

Patients and Methods: Fifty-five human ccRCC tissue samples, ten adjacent nonmalignant kidney tissue samples, 20 paired paraffin section tissues and seven RCC cell lines were obtained. Immunohistochemistry (IHC) and real-time PCR were used to examine the expression of the target genes at the mRNA and protein levels. The methylation of SOX17 was analyzed using methylation-specific PCR (MSP) and bisulfite genomic sequencing (BGS) assay. The functions of SOX17 were examined by using CCK8, colony formation, wound healing assay and Matrigel invasion assays. Luciferase assay was used to analyze the function of SOX17 in the WNT signaling pathway.

Results: We investigated the SOX17 expression in ccRCC tissues and adjacent non-malignant kidney tissues using PCR and IHC. The expression of SOX17 was lower in ccRCC tissues. Next, we analyzed the DNA promoter methylation of SOX17 in 55 human ccRCC tissues, 10 adjacent non-malignant kidney tissues and RCC cell lines using MSP. DNA methylation of the SOX17 promoter region occurred in $60 \%$ of ccRCC tissues and $10 \%$ of adjacent non-malignant kidney tissues. In vitro experiments showed that SOX17 suppressed the proliferation of RCC cells. Furthermore, SOX17 inhibited the migration of RCC cells as shown in the wound healing and migration assays. In addition, we found that SOX17 overexpression affected the WNT signaling pathway by downregulating c-myc and cyclinD1.

Conclusion: In summary, our study showed that SOX17 is downregulated in ccRCC and the loss of SOX17 expression is regulated via epigenetic mechanisms in ccRCC. In addition, SOX17 negatively regulates the WNT signaling pathway and function as a tumor suppressor in ccRCC.

Keywords: ccRCC, SOX17, DNA methylation, WNT signaling pathway

\section{Introduction}

Renal cell carcinoma (RCC) is the major primary malignant tumor in the genitourinary system, accounting for $2-3 \%$ of all malignancies. The latest cancer statistics report showed that nearly 73,820 new RCC cases are diagnosed in the U.S.A, and almost 14,700 patients die every year due to RCC, making it the sixth most common tumor in males. ${ }^{1}$ Clear-cell renal cell carcinoma (ccRCC) is the most common subtype of RCC (85\%). Approximately $16 \%$ of patients have metastasis at the time of diagnosis, and the 5-year relative survival rate is only $12 \%$. Although 
treatment methods for ccRCC have been advanced with the developments in medical oncology and surgery over the last few decades. Unfortunately, the prognosis and outcome of metastatic renal cell carcinoma (mRCC) remain dismal. Nearly 20 to $30 \%$ of patients with ccRCC experience recurrence after treatment, and no approved therapy exists to reduce the risk of recurrence, progression, or death. ${ }^{2,3}$ Recently, target therapy has been proven to prolong the survival of patients' with $\mathrm{mRCC}$; however, the median survival is still less than 3 years. ${ }^{4}$ Furthermore, drug resistance and financial burden are problems in clinical practice. ${ }^{5}$ Thus, it is necessary to explore the molecular mechanism underlying the pathogenesis of ccRCC.

In recent years, it has been reported that tumorigenesis is caused by increased levels of genetic and epigenetic alterations that ultimately convert normal cells into cancer cells. DNA methylation is one of the most frequent epigenetic events in humans correlated with carcinogenesis. The alterations in DNA methylation are quite common in cancer cells, including ccRCC cells. ${ }^{4}$ It is well established that aberrant methylation of $\mathrm{CpG}$ islands results in the loss of expression of tumor suppressor genes. ${ }^{5}$ Aberrant methylation of tumor suppressor genes appears to be an early event that plays a significant role in the development and progression of cancer. ${ }^{6}$ In addition, based on the analysis of the DNA methylation profile, epigenetic biomarkers have proven to be useful in the diagnosis and prognostication of various tumors. ${ }^{7,8}$ Therefore, it is necessary to increase scientific research to identify DNA-specific methylation profile, and find new strategies for the early diagnosis, prognostic evaluation, and treatment of ccRCC.

SOX17, a member of the SRY-related high mobility group box (Sox) family transcription factors, is conserved in many species. According to the classification of SOX genes, SOX17 belongs to the SOX group F. ${ }^{9}$ SOX $\mathrm{F}$ proteins, including SOX7, SOX17, and SOX18, are characterized by a distinct $\mathrm{C}$-terminal transactivation domain. The SOX F members also contain a short amino acid motif (DXXEFD/EQYL) within the transactivation domain, mediating $\beta$-catenin interactions. SOX17 is implicated in various biological activities and developmental processes, such as the formation of the endoderm, oligodendrocyte development and embryonic hematopoiesis. $^{10,11}$ Furthermore, SOX17 has also been reported to participate in the regulation of stem cell formation, and it can function as a negative regulator of $\beta$ catenin/TCF transcription in the WNT signal transduction pathway. $^{12}$ Growing evidence suggests that SOX17 may also play a role in the carcinogenesis of gastric cancer, hepatocellular carcinoma, and breast cancer in humans. ${ }^{13-15}$ It has been reported that SOX17 is a tumor suppressor that inhibits the canonical WNT/ $\beta$-catenin signaling pathway in thyroid cancer. ${ }^{16}$ In addition, it has been reported that SOX17 was downregulated upon promoter methylation in breast cancer. Downregulation of SOX17 upregulates the expression of $\beta$-catenin. This study demonstrated that hypermethylation of the SOX17 promoter contributes to the aberrant activation of the WNT signaling pathway. ${ }^{17}$ However, the regulatory function of SOX17 in ccRCC is insufficiently understood and the molecular mechanisms underlying the role of SOX17 in ccRCC development and progression are largely unknown.

The WNT signaling pathway is a highly conserved pathway and plays significant roles in both physiological and pathophysiological processes, including cell differentiation, migration and proliferation during embryonic development and adult homeostasis. ${ }^{18,19}$ Additionally, increasing evidence has shown that the aberrant activation of the WNT signaling pathway may contribute to various types of diseases and human cancers. ${ }^{19,20}$ It has also been confirmed that dysregulation of the WNT signaling pathway is associated with RCC progression. ${ }^{21}$ However, the mechanisms underlying these phenomena have not been well understood. Recently, it has been reported that many WNT pathway antagonists, such as SFRP, ${ }^{22}$ SOX7, ${ }^{23}$ $\mathrm{DKK}^{24}$ are epigenetically silenced in RCC, indicating that epigenetic silencing of WNT inhibitors may present an important mechanism promoting the development of RCC.

In light of these facts, this study aimed to investigate the methylation of SOX17 in ccRCC and analyze the relationship between SOX17 methylation and clinical features of ccRCC. Furthermore, we ought to explore the function of SOX17 in the tumorigenesis of ccRCC.

\section{Materials and Methods}

\section{Cell Lines and Human Tissue Specimens}

Seven RCC tumor cell lines and two normal control cell lines (786-O, A498, CAKI1, CAKI2, OSRC, 769P, Koto3, HEK293T, and HK-2) were purchased from American type culture collection (ATCC, Manassas, VA). Human normal embryonic kidney cell line (HEK293T) and normal human proximal tubular cell line $(\mathrm{HK}-2)$ were used as a normal controls. RCC samples (55 fresh RCC tissues, 10 adjacent non-malignant fresh tissues, and 20 paired 
paraffin-embedded tissue sections) in our study were collected from the Department of Urology, Shengjing Hospital of Chinese Medical University, Shenyang, People's Republic of China since 2017 to 2019. The protocol of our study was approved by the institutional reviewer board of the Shengjing Hospital. The samples in this study were from patients who had underwent primary radical surgical or partial nephrectomy without adjuvant therapy history and with prior history of RCC. Written informed consent was provided by the patients and this was conducted following the Declaration of Helsinki (Approval No.: 2020PS362K(X1)), approved on April 20, 2020. In addition, all these samples were diagnosed by two urological pathologists. Fuhrman nuclear grade and 2018 American Joint Committee on Cancer (AJCC) TNM stage were used for the tumor histopathology classification. ${ }^{25}$

\section{Data Collection}

To analyze SOX17 expression and promoter methylation levels, we used the Kidney Renal Clear Cell carcinoma cohort on the MethHC database. All data used in our research are open to the public without limitations or restrictions. The expression of SOX17 and Kaplan-Meier overall survival (OS) curves were analyzed according to the data provided in the GEPIA (http://gepia.cancer-pku. $\underline{\mathrm{cn}})$. The expression of SOX17 at different stages of ccRCC was downloaded from The Cancer Genome Atlas portal (TCGAportal, http://tumorsurvival.org/).

\section{Cell Culture and Demethylation Treatment}

All the cell lines were incubated at $37^{\circ} \mathrm{C}$ in a humidified atmosphere containing $5 \% \mathrm{CO}_{2}$ and routinely maintained in Roswell Park Memorial Institute (RPMI-1640) or Dulbecco's modification of Eagle's medium Dulbecco (DMEM) supplemented with $10 \%$ fetal bovine serum (Thermo Fisher Scientific, Waltham, MA, USA), $100 \mathrm{U} /$ $\mathrm{mL}$ penicillin $\mathrm{G}$, and $100 \mathrm{mg} / \mathrm{mL}$ streptomycin. RCC cells were seeded at a density of $1 \times 10^{6}$ cells $/ \mathrm{mL}$ onto a six-well plate, and after being cultured for $12 \mathrm{~h}$, they were treated with $10 \mu \mathrm{M}$ of the demethylation agent 5-Aza (5-aza-2'deoxycytidine) for 96 hours, and then with $100 \mathrm{nmol} / \mathrm{L}$ of the histone deacetylase inhibitor trichostatin A (TSA) for an additional 24 hours. The cells were collected for DNA and RNA extraction.

\section{DNA and RNA Extraction}

Total RNA was extracted from RCC cell lines and the clinical primary tissues, using the TRI Reagent and Tianamp Genomic DNA Kits (TIANGEN ${ }^{\circledR}$, Shanghai, China) as described in our previous study. ${ }^{26}$ Genomic DNA of primary RCC tissues and RCC cells was isolated using TIANamp Genomic DNA Kits (TIANGEN $^{\circledR}$, Shanghai, China) according to the manufacturer's instructions. DNA and RNA concentrations were assessed using the ND2000 Spectrophotometer (Thermo Fisher Scientific).

\section{SOX 17-Expressing Vector and Infection}

The pLVX-IRES-ZsGreen1-SOX17 vector containing human full-length SOX17 cDNA and the negativecontrol vector pLVX-IRES-ZsGreen1 were obtained from YouBio company (Hunan, China). The SOX17 cDNA sequence was confirmed via DNA sequencing. Then, 786-O and A498 cells were infected with the pLVXIRES-ZsGreen1-SOX17 and pLVX-IRES-ZsGreen1 vectors following the manufacturer's instructions.

\section{RT-PCR and Real-Time PCR}

RNA expression was detected using the GoTaq Green Master Mix (Promega, Madison, WI, USA) and ABI Prism 7500 ${ }^{\mathrm{TM}}$ instrument (Applied Biosystems) with SYBR Green PCR Mix. Primers are listed in Table $1 .^{14,27}$ Exactly $1 \mu \mathrm{L}$ of cDNA was added to $12.5 \mu \mathrm{L}$ of the PCR reaction. A total of 32 cycles $\left(94^{\circ} \mathrm{C}\right.$ for $30 \mathrm{~s}, 55^{\circ} \mathrm{C}$ for $30 \mathrm{~s}$ and $72^{\circ} \mathrm{C}$ for $\left.30 \mathrm{~s}\right)$ of amplification were performed for each RT-PCR reaction. Then, the amplified products were analyzed on $1.5 \%$ agarose gels. Real-time PCR was performed for 45 cycles $\left(94^{\circ} \mathrm{C}\right.$ for $30 \mathrm{~s}, 55^{\circ} \mathrm{C}$ for $30 \mathrm{~s}$ and $72^{\circ} \mathrm{C}$ for $30 \mathrm{~s}$ ); glyceraldehyde3-phosphate dehydrogenase (GAPDH) was used as an internal control. All assays were repeated at least three times.

\section{DNA Bisulfite Modification and Promoter Methylation Analysis}

DNA of RCC cells and primary RCC tissues were exposed to sodium bisulfite modification using the EpiTect Bisulfite Kit (Qiagen, Hilden, Germany), following the manufacturer's instructions. Then, bisulfite DNA was used for Methylation specific PCR (MSP) and Bisulfite Genomic Sequencing (BGS) to identify SOX17 promoter methylation status. MSP was performed using a reaction volume of $12.5 \mu \mathrm{L}$ using the GoTaq1 Green Master Mix (Promega, Madison, WI,USA) for 36 cycles $\left(94^{\circ} \mathrm{C}\right.$ for $30 \mathrm{~s}, 56^{\circ} \mathrm{C}$ for $30 \mathrm{~s}$, and $72^{\circ} \mathrm{C}$ for $30 \mathrm{~s}$ ). The MSP products were further 
Table I Primers Used in the Research

\begin{tabular}{|c|c|c|}
\hline Type & Primers & Sequence $\left(5^{\prime}-3^{\prime}\right)$ \\
\hline \multirow[t]{10}{*}{ RT-PCR } & SOXI7-R & AGC CCT GCT CGG GGA ACT \\
\hline & SOXI7-F & GGC TGG CGC AGC AGA ATC \\
\hline & MMP2-R & GATACCCCTTTGACGGTAAGGA \\
\hline & MMP2-F & ССTTCTCCCAAGGTCCATAGC \\
\hline & MMP9-R & GGGACGCAGACATCGTCATC \\
\hline & MMP9-F & TCGTCATCGTCGAAATGGGC \\
\hline & c-myc-F & GTCAAGAGGCGAACACACAAC \\
\hline & c-myc-R & TTGGACGGACAGGATGTATGC \\
\hline & CyclinDI-F & GCTGCGAAGTGGAAACCATC \\
\hline & CyclinDI-R & ССТССTTCTGCACACATTTGAA \\
\hline \multirow[t]{4}{*}{ MSP } & SOXI7-MR & $\begin{array}{l}\text { GGG GCG TTC GTA GTG TTA TTA } \\
\text { GGT C }\end{array}$ \\
\hline & SOXI7-MF & $\begin{array}{l}\text { AAA CAC TAA AAT ACC CCG AAA } \\
\text { ACT ACG }\end{array}$ \\
\hline & SOXI7-UR & $\begin{array}{l}\text { TTA GGG GTG TTT GTA GTG TTA } \\
\text { TTA GGT T }\end{array}$ \\
\hline & SOXI7-UF & $\begin{array}{l}\text { TAA AAC ACT AAA ATA CCC CAA } \\
\text { AAA CTA CA }\end{array}$ \\
\hline \multirow[t]{2}{*}{ BGS } & SOXI7-BF & $\begin{array}{l}\text { GTT TTA TTG GTT ATA TTT GTG } \\
\text { TAG }\end{array}$ \\
\hline & SOXI7-BR & ACT ACT CAT AAC RCT CCA AAC \\
\hline
\end{tabular}

analyzed using $2 \%$ agarose gel electrophoresis. For BGS, PCR products were amplified using BGS primers and then cloned into the pEASY-T5 zero vectors (TransGen Biotech Co. Ltd., Beijing). Six to ten colonies were randomly selected and sequenced via BGS. All the primers used are listed in Table 1.

\section{Cell Proliferation Assay}

Cells infected with the SOX17-expressing vector and control vector were seeded onto a 96-well plate at a density of 2000 cells/well using CCK-8 (Dojindo, Kumamoto,Japan) according to the manufacturer's instructions. The absorbance of the samples were measured at $450 \mathrm{~nm}$. All experiments were repeated three times.

\section{Colony Formation Assay}

Colony formation assay was used to analyze the proliferative ability of RCC cells. pLVX-IRES-ZsGreen1-SOX17 and pLVX-IRES-ZsGreen1 infected cells were plated at a density of 400, 800 and 1200 cells/well in a six-well plate and cultured for 10-14 days. Then, the cells were fixed with $75 \%$ ethanol for $40 \mathrm{~min}$, and $0.2 \%$ crystal violet (Beyotime, Nanjing, China) was used to stain the cells for $40 \mathrm{~min}$. Then, the number of colonies formed ( $>50$ cells/ colony) were counted.

\section{Wound Healing Assay}

For the wound-healing assay, pLVX-IRES-ZsGreen1SOX17 and pLVX-IRES-ZsGreen1 infected cells were seeded onto six-well culture plates and cultured to $90-95 \%$ confluency. Then, a scratch wound was created in the cell monolayer. Images were captured at 0, 12, 24 and $48 \mathrm{~h}$.

\section{Matrigel Invasion Assay}

The cell invasion assay was performed using a Transwell chamber containing $20 \mu \mathrm{L}$ of Matrigel (BD Bioscience; dilution, $1: 4$ ). Precisely $2 \times 10^{5}$ cells were suspended in 200 $\mu \mathrm{L}$ of serum-free medium and transferred to the upper chamber. After $24 \mathrm{~h}$ of incubation, the cells in the upper chambers were removed and the cells passing through the filter were fixed with methanol for $25 \mathrm{~min}$ and stained with crystal violet. The cells were counted using a microscope set. All assays were independently repeated at least three times.

\section{Luciferase Assay}

The cells were plated at a density of $2 \times 10^{4}$ cells/well onto 24-well plates and cultured $24 \mathrm{~h}$ before transfection. Then, the cells were lysed and luciferase activity was measured using the dual-luciferase reporter assay (Promega, Madison, WI) on a GloMax luminometer (Promega\#SA3030). The relative luciferase activity was presented as the luciferase activity of the Firefly target pGL3 reporters and was normalized to the pRL-TK Renilla luciferase activity.

\section{Immunohistochemistry (IHC)}

The IHC analysis was performed on 20 paired paraffinembedded RCC tissues and adjacent non-malignant tissues using the primary anti-SOX17 antibody (09-038-I MSDS; Sigma-Aldrich). The paraffin-embedded tissues were boiled with $10 \mathrm{mM}$ citrate buffer ( $\mathrm{pH}$ 6.0) for 2 min for antigen retrieval. Then, the sections were washed three times in PBS and permeabilized with $0.1 \%$ Triton X-100. Subsequently, they were exposed to the blocking solution (PBS/3\% hydrogen peroxide) and incubated with the antiSOX17 antibody overnight at $4{ }^{\circ} \mathrm{C}$. After three washes with 
PBS, the sections were incubated with secondary antibodies for $30 \mathrm{~min}$ at room temperature and counterstained with hematoxylin. The images were captured and analyzed using the Image Pro Plus software (IPP, Version 6.0, Media Cybernetics, Silver Spring, MD, USA).

\section{Western Blotting}

Total protein was extracted using the Keygen Biotech protein extraction kit (KGP1100, KGP150; Nanjing, China). Equal amounts of the proteins were separated using $10 \%$ SDS-PAGE, then, they were transferred onto nitrocellulose membranes. After blocking with $5 \%$ fat-free milk at $4^{\circ} \mathrm{C}$ overnight, immunoblotting was performed with the following specific primary antibodies; AntiSOX17 (09-038-I MSDS; Sigma-Aldrich), anti- $\beta$-catenin (8480P; Cell Signaling Technology), anti-c-myc (5605P; Cell Signaling Technology), and anti-cyclinD1 (2926P; Cell Signaling Technology).

\section{Statistical Analysis}

Two-tailed $t$-tests, Fisher's exact test, and $x^{2}$ tests were used to analyze the results. We used the SAS software (v.9.2; SAS Institute, Cary, NC, USA) to calculate the correlation between clinicopathologic features and methylation. The correlation between SOX17 and ccRCC stage was analyzed using the Wilcoxon signed-test. All the results were expressed as the means \pm standard deviation. Differences with $p$ values $<0.05$ were considered statistically significant.

\section{Results}

\section{Downregulation of SOXI7 in Primary RCC Samples}

The SOX17 expression levels in primary RCC tissues, compared with those in normal kidney tissues, were obtained from GEPIA (Figure 1A). Downregulation of SOX17 was statistically significant in renal papillary cell carcinoma (KIRP) and renal chromophobe cell carcinoma (KICH), no statistical significance was found in clear cell renal cell carcinoma (KIRC). In addition, the database also showed a significant difference of OS curves between high SOX17 expression cluster and low SOX17 expression cluster (Figure $1 \mathrm{~B} ; \mathrm{p}<0.05)$. To further validate these findings, the expression of SOX17 in 12 paired RCC tumors and adjacent nonmalignant tissues was analyzed using real-time PCR. SOX17 was weakly expressed in 12/12 primary RCC tissues compared with that in the paired adjacent non-malignant kidney tissues (Figure 1C, p <0.05). Moreover, we used IHC to analyze SOX17 expression in 20 primary RCC tumors and paired adjacent non-malignant tissues. The IHC results
A
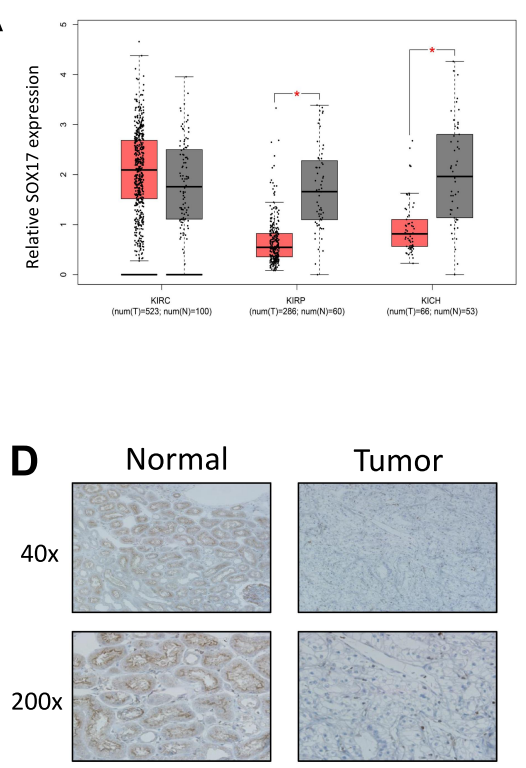

B $_{-}$

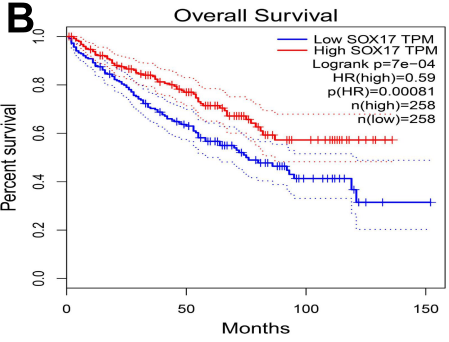

E

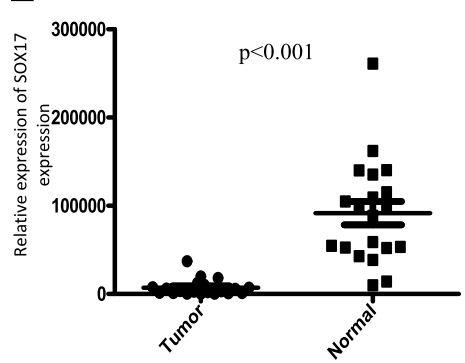

C

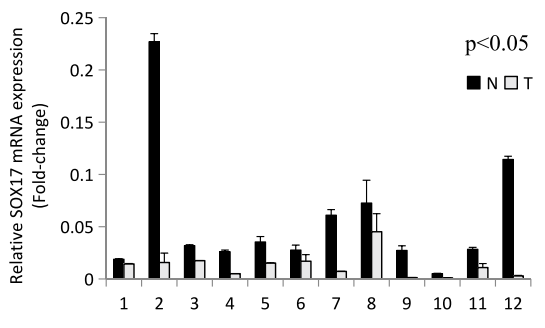

F

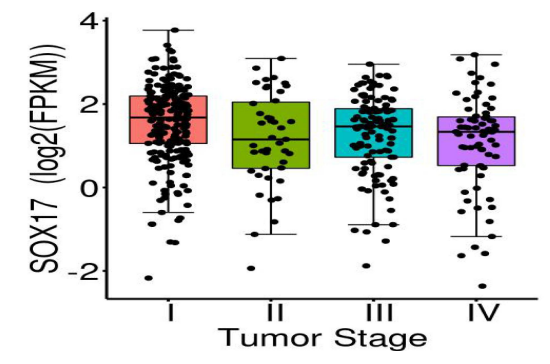

Figure I SOXI7 expression is decreased in primary RCC tissues compared with that in adjacent non-malignant kidney tissues. (A) Expression of SOXI7 in RCC tissues and normal tissues. *p<0.0I. (http://gepia.cancer-pku.cn); (B) Kaplan-Meier overall survival (OS) curve for patients with ccRCC between the two clusters. (C) Real-time PCR revealed SOX17 mRNA expression levels in 12 paired RCC tissues, N (black columns): adjacent non-malignant renal tissues, T (empty columns): primary RCC tissues, $\mathrm{P}<0.05$. (D) Immunohistochemistry staining showed SOXI7 expression in 20 paired RCC samples at $40 \times$ and $200 \times$ magnification. (E) Image Pro Plus software was used to analyze the SOX17 quantitative protein expression; $\mathrm{p}<0.00 \mathrm{I}$. (F) SOXI7 mRNA expression at different stages of ccRCC (http://tumorsurvival.org/). 
showed that the expression of SOX17 was decreased in 20/20 RCC primary RCC tissues, compared with that in corresponding adjacent non-malignant kidney tissues (Figure 1D). We then used the Image Pro-Plus software to analyze SOX17 expression and found a significant difference between the SOX17 expression in RCC tumors and adjacent non-malignant tissues (Figure $1 \mathrm{E}, \mathrm{p}<0.01$ ). We observed a loss of SOX17 expression at both the mRNA and protein levels. The TCGAportal demonstrated a correlation between SOX17 expression and ccRCC stage (Figure 1F). The corresponding statistical results are shown in Table 2.

\section{Epigenetic Silencing of SOXI7 in RCC Cell Lines}

In RCC cell lines, RT-PCR was employed to examine the expression of SOX17. The result showed that SOX17 was weakly expressed in the CAKI-2 cell line, and no expression was observed in the 786-O, A498, CAKI-1, OSRC, 769P and KOTO-3 cell lines. However, in two "normal" kidney cell lines (HK-2 and HEK-293), SOX17 was robustly expressed (Figure 2A). Aberrant promoter methylation of SOX17 was observed in 4/5 RCC cell lines (786-0, CAKI-1, CAKI-2 and OSRC) using MSP (Figure 2B). We found that in the presence of demethylation agents
Table 2 Correlation Between SOXI7 Expression and ccRCC Stage

\begin{tabular}{|l|c|c|c|c|}
\hline & Stage I & Stage II & Stage III & Stage IV \\
\hline Stage I & I & 0.048 & 0.018 & 0.002 \\
Stage II & 0.048 & I & 0.565 & 0.857 \\
Stage III & 0.018 & 0.565 & I & 0.201 \\
Stage IV & 0.002 & 0.857 & 0.201 & I \\
\hline
\end{tabular}

(5-aza-2'-deoxycytidine, either alone or in combination with TSA), SOX17 was upregulated accompanied with the decreased methylation status or increased unmethylation of alleles (Figure 2A and B). Moreover, a high density of methylated alleles was observed in 786-O cells using BGS. However, after treatment with demethylation agents, the 786-O cells showed a low density of methylated alleles (Figure 2C).

\section{Hypermethylation of the SOXI7 Promoter in Primary RCC Samples}

As demonstrated in the MethHC database, higher SOX17 methylation was observed in RCC tissues than in normal tissues (Figure $3 \mathrm{~A}, \mathrm{p}<0.005$ ). Furthermore, the database also showed a negative correlation between SOX17 expression and the methylation of its promoter
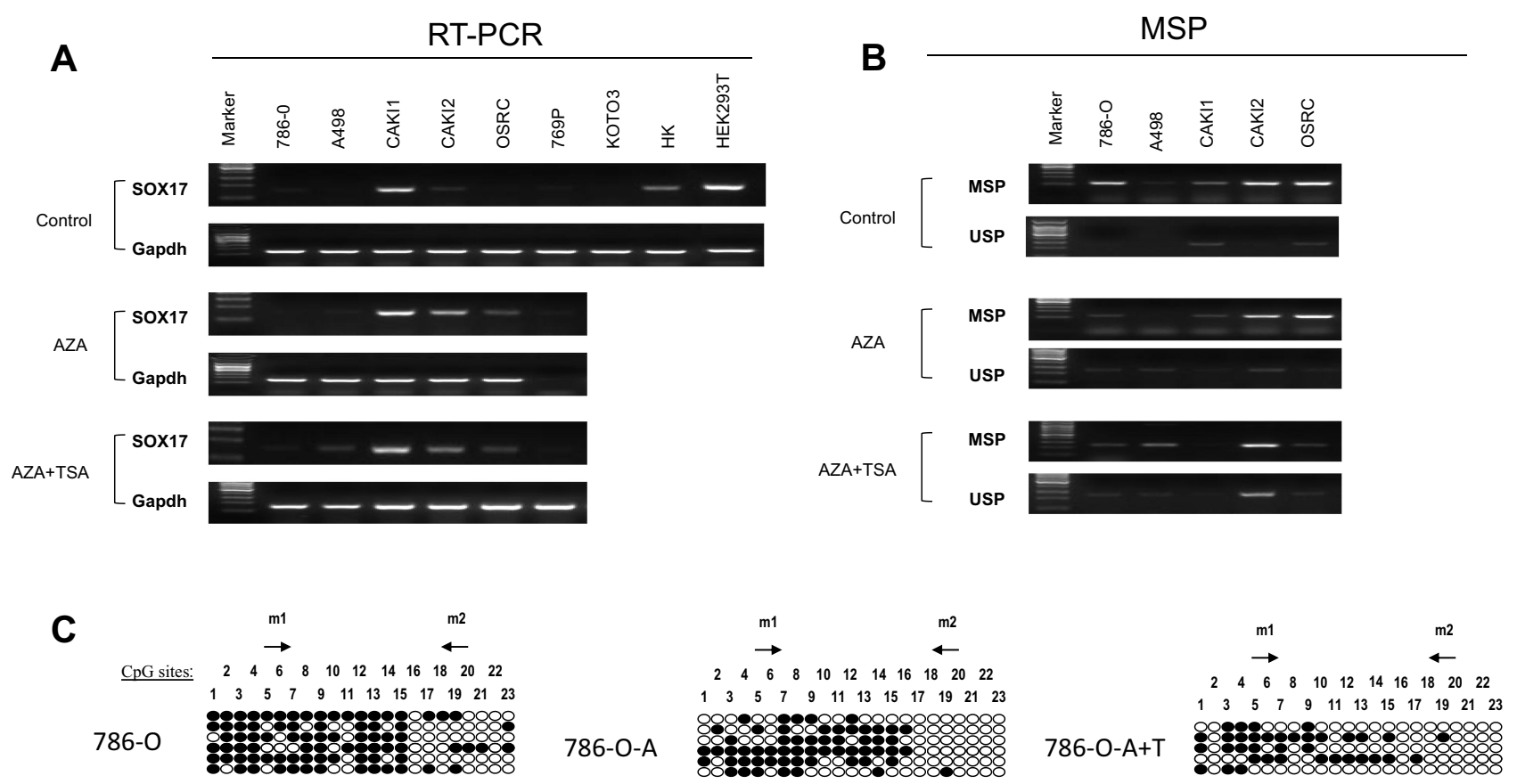

Figure 2 SOXI7 is predominantly silenced/downregulated via promoter CPG methylation in RCC. (A) Demethylation treatment with 5-aza-2' deoxycytidine alone or combined with TSA (A+T) restored SOXI7 expression in RCC cell lines; GAPDH was used as an internal control. (B) After pharmacologic demethylation, the SOXI7 methylation level decreased in methylated RCC cell lines, as demonstrated using MSP. M: methylation, U: unmethylation. (C) BGS analysis of SOXI7 promoter region in $786-$ $O$ cells after demethylation treatment. The filled circles represent methylated $C_{p} G$ sites, and the open circles represent unmethylated $C_{p} G$ sites. 
A

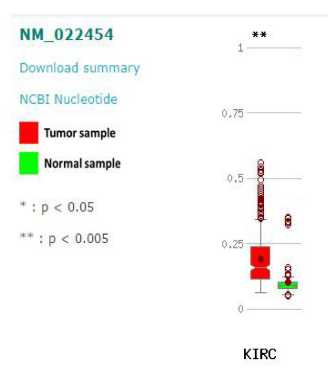

B

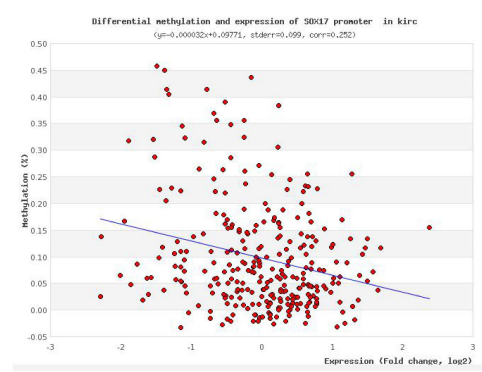

C
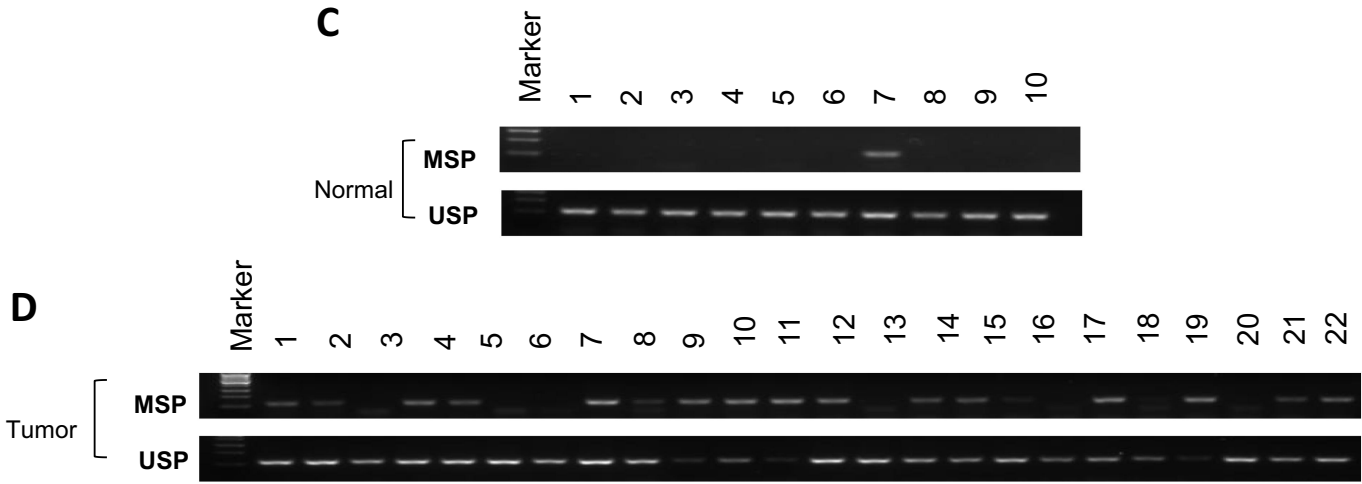

E
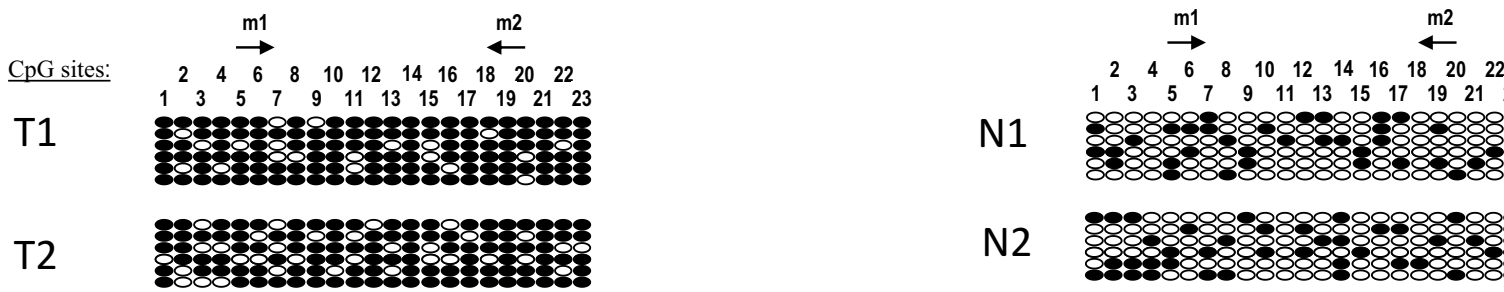

N1 $\begin{array}{llllllllllll}1 & 3 & 5 & 7 & 9 & 11 & 13 & 15 & 17 & 19 & 21 & 23\end{array}$

CpG sites: $\begin{array}{llllllllllll}1 & 3 & 5 & 7 & 9 & 11 & 13 & 15 & 17 & 19 & 21 & 23\end{array}$

Figure 3 Methylation level of SOXI7 was higher in primary RCC tissues than in normal kidney tissues. (A) SOXI7 methylation was higher in RCC tissues than in normal kidney tissues, as obtained from PubMed Database, ${ }^{* *} \mathrm{p}<0.005$. (B) Methylation and expression of SOXI7 were negatively correlated in RCC samples. One red circle represents one clinical sample. (C) SOXI7 methylation status in adjacent non-malignant kidney tissues, determined using MSP. (D) SOXI7 methylation in RCC tissues, determined using MSP. (E) Methylation of SOXI7 promoter in human tissues was shown using BGS.

(Figure 3B). These results suggested that SOX17 was often hypermethylated in RCC tumors. We then used MSP and BGS to detect SOX17 methylation in primary RCC tumors and adjacent non-malignant tissues. The methylation level of SOX17 in primary RCC tumors was $60 \%(33 / 55)$, however, only $10 \%(1 / 10)$ of SOX17 methylation was detected in adjacent non-malignant tissues (Table 4), as assessed using MSP (Figure 3C and D). To verify SOX17 promoter methylation, BGS was performed. The results demonstrated that SOX17 methylation was higher in RCC tumors than in adjacent nonmalignant kidney tissues (Figure 3E). We also examined the association of SOX17 methylation with clinicopathological features of patients with RCC. No significant correlation between SOX17 methylation and clinical features was found in this cohort (Table 3).

\section{SOXI7 Suppresses RCC Cell Proliferation}

Epigenetic silencing of SOX17 in RCC tumors and cell lines indicated that it might function as a tumor suppressor in RCC. Thus, we examined its function in two SOX17deficient cell lines (786-O and A498). These two cell lines were first infected with pLVX-IRES-ZsGreen1 and pLVXIRES-ZsGreen1-SOX17 vectors, and the expression of SOX17 was detected using PCR (Figure 4A). The CCK8 assay showed that SOX17 strongly inhibited RCC cell proliferation (Figure 4B). To further evaluate the effect of SOX17 on cell viability, we performed colony formation assay. The results of the colony formation assay showed that the ectopic expression of SOX17 significantly suppressed the numbers of cell colonies, compared to the case for the control cells (Figure 4C). 
Table 3 Correlation of SOXI7 Methylation and Clinicopathological Features in RCC

\begin{tabular}{|c|c|c|c|c|}
\hline \multicolumn{2}{|c|}{ Clinicopathological Feature } & Methylated No. (\%) & Unmethylated No. (\%) & p value \\
\hline Age & & $57.34+13.09$ & $55.45+11.9$ & 0.52 \\
\hline Gender & $\begin{array}{l}\text { Male } \\
\text { Female }\end{array}$ & $\begin{array}{l}20(62.5 \%) \\
13(56.5 \%)\end{array}$ & $\begin{array}{l}12(37.5 \%) \\
10(43.5 \%)\end{array}$ & 0.65 \\
\hline Side & $\begin{array}{l}\text { Left } \\
\text { Right }\end{array}$ & $\begin{array}{l}18(69 \%) \\
15(51.7 \%)\end{array}$ & $\begin{array}{l}8(3 \mid \%) \\
\mid 4(48.3 \%)\end{array}$ & 0.18 \\
\hline TNM classification & $\begin{array}{l}\text { PTI } \\
\text { PT2 } \\
\text { PT3 } \\
\text { PT4 }\end{array}$ & $\begin{array}{l}23(69.7 \%) \\
5(45.5 \%) \\
4(40 \%) \\
1(100 \%)\end{array}$ & $\begin{array}{l}10(30.3 \%) \\
6(54.5 \%) \\
6(60 \%) \\
0(0)\end{array}$ & 0.15 \\
\hline Nuclear grade & $\begin{array}{l}\mathrm{GI} \\
\mathrm{G} 2 \\
\mathrm{G} 3\end{array}$ & $\begin{array}{l}21(53.8 \%) \\
8(72.7 \%) \\
4(80 \%)\end{array}$ & $\begin{array}{l}\mathrm{I} 8(46.2 \%) \\
3(27.3 \%) \\
\mathrm{I}(20 \%)\end{array}$ & 0.15 \\
\hline
\end{tabular}

\section{SOXI7 Inhibits RCC Cell Migration and Invasion}

To analyze the effect of SOX17 on invasion and migration, wound-healing and Transwell assays were performed. In the Transwell assay, SOX17-expressing cells displayed a significantly decreased invasiveness ability compared to the control cells (Figure 5A). Furthermore, SOX17 decreased the matrix metallopeptidase (MMP) 2 and 9 expression levels in 786-O and A498 cells (Figure 5B). As shown in the wound healing assay results, SOX17expressing cells were less efficient in closing an artificial wound than the cells infected with the empty vector on the confluent monolayer (Figure $5 \mathrm{C}, \mathrm{p}<0.05$ ). These results indicated that SOX17 suppressed the RCC cells migration and invasion.

\section{SOXI7 Antagonizes the WNT Signaling Pathway}

To explore the effect of SOX17 on the WNT signaling pathway in RCC and detect the expression of WNT signaling pathway downstream components (c-myc and cyclin D1), real-time PCR was employed. The expression levels of

Table 4 Methylation Status of SOXI7 in Primary RCC Samples

\begin{tabular}{|l|l|l|l|}
\hline \multirow{2}{*}{$\begin{array}{l}\text { RCC } \\
\text { Samples }\end{array}$} & \multicolumn{2}{|c|}{ CCND2 Promoter } & Methylation \\
\cline { 2 - 3 } & Methylation & Unmethylation & \\
\hline Tumor & 33 & 22 & $60 \%$ \\
Normal & 1 & 9 & $10 \%$ \\
\hline
\end{tabular}

c-myc and cyclinD1 were reduced in SOX17-expressing cells, compared with those in control cells (Figure 6A). Furthermore, luciferase activity was decreased in cells infected with SOX17 compared to the cells infected with the empty vector (Figure 6B). Ectopic expression of SOX17 reduced the $\beta$-catenin, c-myc and cyclinD1 expression levels (Figure 6C). These results demonstrated that the transcriptional activity of WNT signaling pathway was suppressed by SOX17.

\section{Discussion}

In our study, we demonstrated that SOX17 played a significant role as a tumor suppressor in ccRCC through a comprehensive analysis of SOX17 expression in $\mathrm{RCC}$ cell lines and primary ccRCC tumors. This study showed that SOX17 silencing by promoter methylation frequently occurs in RCC cell lines and primary tumors. It also suggested that promoter methylation was a dominant regulatory mechanism underlying the downregulation of SOX17. Furthermore, we also observed that SOX17 suppressed RCC cells growth and invasiveness and antagonized the WNT signaling pathway. Our results clearly indicated that SOX17 functioned as a tumor suppressor and was epigenetically inactivated in RCC pathogenesis.

Growing evidence has shown that aberrant epigenetic changes in tumor suppressive genes leads to RCC. ${ }^{23,27,28}$ Therefore, identification of epigenetic alterations in RCC could be helpful to unravel the mechanisms underlying RCC carcinogenesis and identify potential biomarkers for cancer screening and prognosis prediction. ${ }^{29}$ SOX17 was reported to be aberrantly methylated in various tumors, 
A

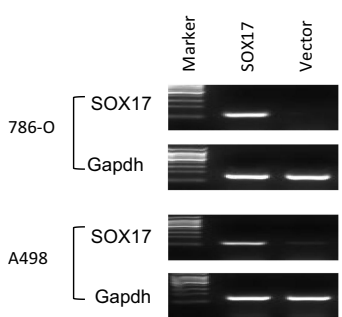

B

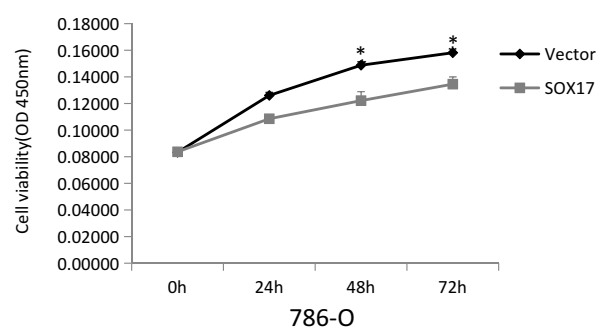

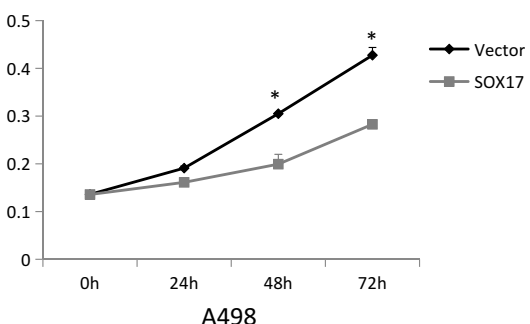

C
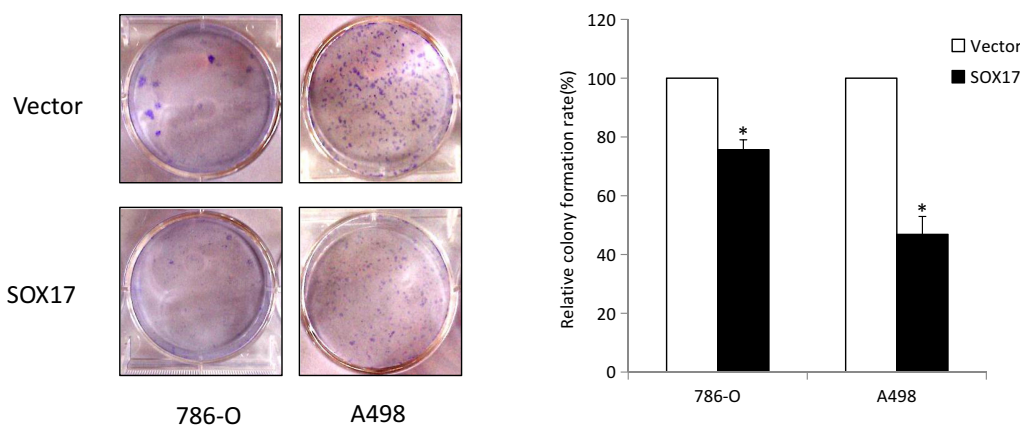

Figure 4 SOXI7 inhibits RCC cell growth. (A) Ectopic expression of SOXI7 was demonstrated using RT-PCR in 786-O and A498 cells after pLVX-IRES-ZsGreen I-SOXI7 or pLVX-IRES-ZsGreen I infection. (B) Cell growth curve showed an inhibitory effect of SOXI7 on cell growth, as determined using the CCK-8 assay, * ${ }^{*}<0.05$. (C) Ectopic expression of SOXI7 suppressed the colony formation ability of RCC cells, the experiment was performed in three replicates and presented as the mean \pm standard deviation, ${ }^{*} \mathrm{p}<0.05$.
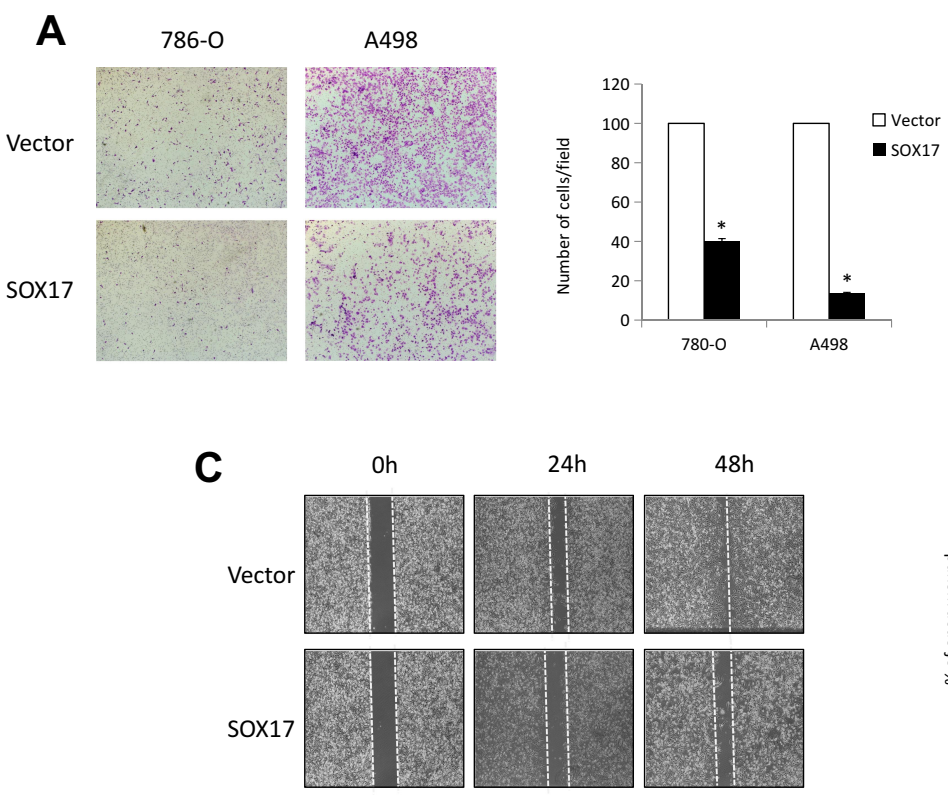

$786-0$
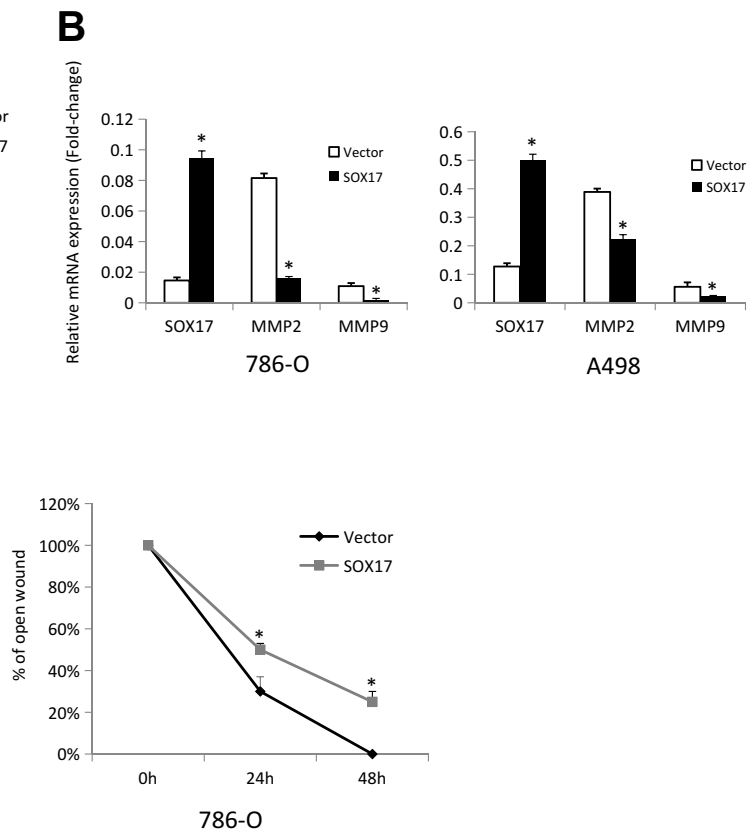

Figure 5 SOXI7 suppresses RCC cell invasion and migration. (A) Ectopic expression of SOXI7 inhibited the migration of 786-O and A498 cells, as shown using the Transwell migration assay. (B) MMP2 and MMP9 expression levels were examined in SOX17-expressing cells and control cells using real-time PCR. (C) The wound healing images of $786-\mathrm{O}$ cells were captured at 0,24 , and $48 \mathrm{~h},{ }^{*} \mathrm{p}<0.05$.

including colon carcinoma, hepatocellular carcinoma and breast carcinoma, ${ }^{12,14,29}$ suggesting the contribution of epigenetic regulation in its inactivation. In our research, we observed that SOX17 was silenced by aberrant promoter methylation, and demethylation treatment restored the expression of SOX17. These results indicated the epigenetic regulation of SOX17 in RCC. In addition, SOX17 is frequently methylated in human primary RCC 


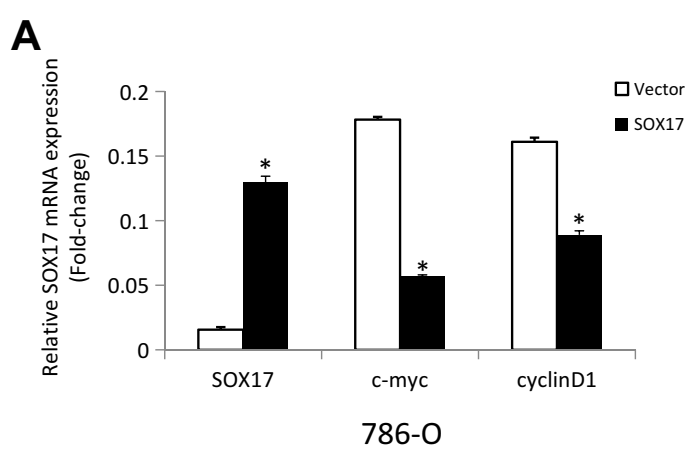

B

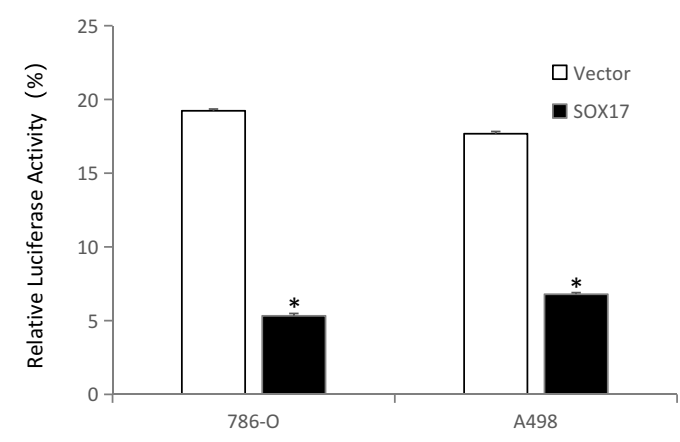

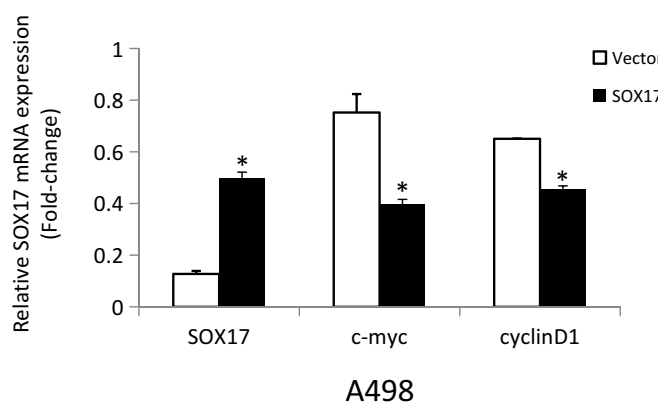

C

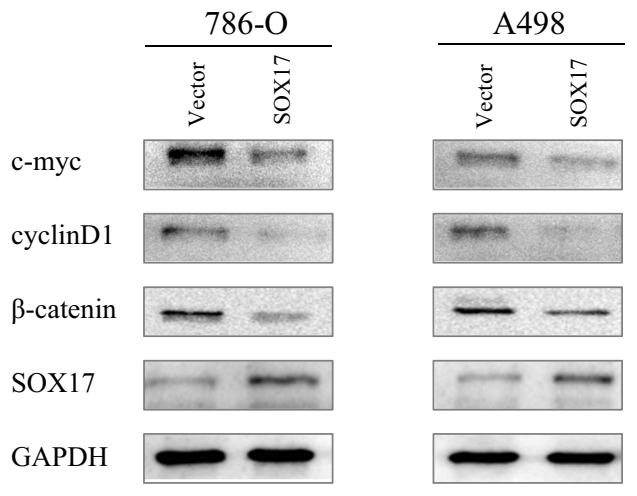

Figure 6 SOXI7 inhibits the WNT signaling pathway. (A) Decreased expression of WNT signaling pathway downstream components (c-myc and cyclin DI) in 786-O and A498 cells was detected using real-time PCR, ${ }^{*} p<0.05$. (B) SOXI7 expression decreased the transcription of WNT signaling pathway components in $786-O$ and A498 cells, as determined using the luciferase reporter assay; ${ }^{*} \mathrm{p}<0.05$. (C) Expression of SOXI7, c-myc, cyclin DI, and $\beta$-catenin were estimated using Western blotting.

$(60 \%)$ but only $10 \%$ methylation was found in adjacent non-malignant tissues, indicating that SOX17 methylation may be an epigenetic biomarker for RCC. In addition, we found that SOX17 suppressed RCC cells proliferation and inhibited RCC cells migration, suggesting that it functioned as a tumor suppressor in RCC. A recent study has reported that downregulation of $\mathrm{SOX} 17$ facilitates the targeted drug resistance and progression of clear cell renal cell carcinoma (ccRCC), which suggests SOX17 as a potential target for inhibiting targeted drug resistance and metastasis in advanced ccRCC. ${ }^{31}$ According to these results, we proposed that restoring SOX17 expression may present a new strategy for suppressing targeted drug resistance and progression of ccRCC.

Deregulation of the WNT signaling pathway, as shown in several reports, is associated with $\mathrm{RCC}$ carcinogenesis. $^{31}$ In RCC, the WNT signaling pathway is also influenced by altered epigenetic regulation. Many WNT antagonists including SFRP1, SFRP2, DKK1, DKK2 and DKK $3^{32-35}$ are inactivated by promoter methylation, resulting in the suppression of the negative regulation of WNT signaling. It has been reported that SOX17 functions as a WNT signaling antagonist in hepatocellular carcinoma. ${ }^{14}$ In our study, SOX17 inhibited the transcriptional activation of WNT signaling, as demonstrated using the luciferase reporter assay. However, the epigenetic inactivation of SOX17 may activate the WNT signaling pathway and promote RCC development.

Early diagnosis is crucial for patients with RCC to improve prognosis. Thus, diagnostic biomarkers could be useful for the non-invasive characterization of small renal masses. The aberrant methylation of several genes (RASSF1A, VHL and SFRP1) has been proposed as an early detection markers for RCC, as these genes are methylated early and frequently in RCC development. $^{36-38}$ Our study discovered that the SOX17 methylation level was higher in tumor tissues than in adjacent non-malignant tissues. This indicated that SOX17 could be a diagnostic biomarker for RCC. Although no statistical relationship was found between SOX17 methylation and clinical features. The reason may be the limited number of RCC patients. We plan to use a larger cohort in future studies to enrich our data. Our team will further explore the relevance of SOX17 methylation for the early diagnosis of RCC from blood or urine, and also validate the effects of SOX17 
demethylation treatment on targeted drug resistance and metastasis in advanced ccRCC.

\section{Conclusion}

In summary, our findings indicate that the inactivation of SOX17 by promoter $\mathrm{CpG}$ aberrant methylation is a frequent event in RCC. In addition, SOX17 inhibit tumor cell proliferation and migration by acting as an antagonist of WNT signaling in RCC. The frequently aberrant methylation of SOX17 might be important in the development and progression of RCC, and might be served as a valuable epigenetic biomarker for RCC early diagnosis, prognosis and treatment. Our results demonstrate the tumor-suppressive role and regulatory mechanism of SOX17 in RCC pathogenesis. Thus providing a new prospect for the early diagnosis and targeted therapy of RCC.

\section{Acknowledgments}

The authors thank the studied patients for their willingness to cooperation with our study. This research was supported in part by the grants from the National Nature Science Foundation of China, 81773108, the Joint plan of research, the development program of Liaoning Province (Grant No. 2020JH 2/10300137) and the 345 Talent Project.

\section{Disclosure}

Dr Xiaonan Chen reports grants from Department of Science and Technology of Liaoning Province, during the conduct of the study. The authors report no other conflicts of interest in this work.

\section{References}

1. Siegel RL, Miller KD, Jemal A. Cancer statistics, 2019. CA Cancer J Clin. 2019;69(1):7-34.

2. Hakimi AA, Chen L, Kim PH, et al. The impact of metformin use on recurrence and cancer-specific survival in clinically localized high-risk renal cell carcinoma. Can Urol Assoc J. 2013;7(11-12):E687-E691. doi:10.5489/cuaj.1447

3. De P, Otterstatter MC, Semenciw R, Ellison LF, Marrett LD, Dryer D. Trends in incidence, mortality, and survival for kidney cancer in Canada, 1986-2007. Cancer Causes Control. 2014;25 (10):1271-1281. doi:10.1007/s10552-014-0427-x

4. Jones PA, Baylin SB. The fundamental role of epigenetic events in cancer. Nat Rev Genet. 2002;3(6):415-428. doi:10.1038/nrg816

5. Bird A. DNA methylation patterns and epigenetic memory. Genes Dev. 2002;16(1):6-21. doi:10.1101/gad.947102

6. Herman JG, Baylin SB. Gene silencing in cancer in association with promoter hypermethylation. $N$ Engl J Med. 2003;349(21):2042-2054. doi:10.1056/NEJMra023075

7. Paluszczak J, Baer-Dubowska W. Epigenetic diagnostics of cancer-the application of DNA methylation markers. J Appl Genet. 2006;47 (4):365-375. doi:10.1007/BF03194647
8. Majchrzak-Celinska A, Paluszczak J, Szalata M, et al. The methylation of a panel of genes differentiates low-grade from high-grade gliomas. Tumour Biol. 2015;36(5):3831-3841. doi:10.1007/s13277014-3025-3

9. Wegner M. From head to toes: the multiple facets of Sox proteins. Nucleic Acids Res. 1999;27(6):1409-1420. doi:10.1093/nar/ 27.6.1409

10. Fu DY, Wang ZM, Wang BL, Shen ZZ, Huang W, Shao ZM. Sox17, the canonical Wnt antagonist, is epigenetically inactivated by promoter methylation in human breast cancer. Breast Cancer Res Treat. 2010;119(3):601-612.

11. Kim I, Saunders TL, Morrison SJ. Sox17 dependence distinguishes the transcriptional regulation of fetal from adult hematopoietic stem cells. Cell. 2007;130(3):470-483. doi:10.1016/j.cell.2007.06.011

12. Sinner D, Kordich JJ, Spence JR, et al. Sox 17 and Sox4 differentially regulate beta-catenin/T-cell factor activity and proliferation of colon carcinoma cells. Mol Cell Biol. 2007;27(22):7802-7815. doi:10.1128/ MCB.02179-06

13. Chimonidou M, Strati A, Malamos N, Georgoulias V, Lianidou ES. SOX17 promoter methylation in circulating tumor cells and matched cell-free DNA isolated from plasma of patients with breast cancer. Clin Chem. 2013;59(1):270-279.

14. Jia Y, Yang Y, Liu S, Herman JG, Lu F, Guo M. SOX17 antagonizes WNT/beta-catenin signaling pathway in hepatocellular carcinoma. Epigenetics. 2010;5(8):743-749. doi:10.4161/epi.5.8.13104

15. Balgkouranidou I, Karayiannakis A, Matthaios D, et al. Assessment of SOX17 DNA methylation in cell free DNA from patients with operable gastric cancer. Association with prognostic variables and survival. Clin Chem Lab Med. 2013;51(7). doi:10.1515/cclm-2012-0320.

16. Li JY, Han C, Zheng LL, Guo MZ. Epigenetic regulation of Wnt signaling pathway gene SRY-related HMG-box 17 in papillary thyroid carcinoma. Chin Med J (Engl). 2012;125(19):3526-3531.

17. Stovall DB, Wan M, Miller LD, et al. The regulation of SOX7 and its tumor suppressive role in breast cancer. Am J Pathol. 2013;183 (5):1645-1653. doi:10.1016/j.ajpath.2013.07.025

18. Logan CY, Nusse R. The Wnt signaling pathway in development and disease. Annu Rev Cell Dev Biol. 2004;20:781-810. doi:10.1146/ annurev.cellbio.20.010403.113126

19. Klaus A, Birchmeier W. Wnt signalling and its impact on development and cancer. Nat Rev Cancer. 2008;8(5):387-398. doi:10.1038/ $\operatorname{nrc} 2389$

20. Batra H, Antony VB. The pleural mesothelium in development and disease. Front Physiol. 2014;5:284. doi:10.3389/fphys.2014.00284

21. Bodnar L, Stec R, Cierniak S, et al. Role of WNT/beta-catenin pathway as potential prognostic and predictive factors in renal cell cancer patients treated with everolimus in the second and subsequent lines. Clin Genitourin Cancer. 2018;16(4):257-265. doi:10.1016/j. clgc.2018.01.008

22. Kawakami K, Yamamura S, Hirata H, et al. Secreted frizzled-related protein-5 is epigenetically downregulated and functions as a tumor suppressor in kidney cancer. Int J Cancer. 2011;128(3):541-550. doi:10.1002/ijc. 25357

23. Wang L, Fan Y, Zhang L, et al. Classic SRY-box protein SOX7 functions as a tumor suppressor regulating WNT signaling and is methylated in renal cell carcinoma. FASEB J. 2018;33(1):254-263. doi:10.1096/fj.201701453RR

24. Ueno K, Hirata H, Majid S, et al. Wnt antagonist DICKKOPF-3 (Dkk-3) induces apoptosis in human renal cell carcinoma. Mol Carcinog. 2011;50(6):449-457. doi:10.1002/mc.20729

25. Taneja K, Williamson SR. Updates in pathologic staging and histologic grading of renal cell carcinoma. Surg Pathol Clin. 2018;11 (4):797-812. doi:10.1016/j.path.2018.07.004

26. Zhang Q, Ying J, Zhang K, et al. Aberrant methylation of the 8p22 tumor suppressor gene DLC1 in renal cell carcinoma. Cancer Lett. 2007;249(2):220-226. doi:10.1016/j.canlet.2006.08.019 
27. Wang L, Cui Y, Sheng J, et al. Epigenetic inactivation of HOXA11, a novel functional tumor suppressor for renal cell carcinoma, is associated with RCC TNM classification. Oncotarget. 2017;8 (13):21861-21870. doi:10.18632/oncotarget.15668

28. Peters I, Eggers H, Atschekzei F, et al. GATA5 CpG island methylation in renal cell cancer: a potential biomarker for metastasis and disease progression. Bju Int. 2012;110(2b):E144-E152. doi:10.1111/ j.1464-410X.2011.10862.x

29. Cancer Genome Atlas Research Network. Comprehensive molecular characterization of clear cell renal cell carcinoma. Nature. 2013;499 (7456):43-49. doi:10.1038/nature12222

30. Fu D, Ren C, Tan H, et al. Sox 17 promoter methylation in plasma DNA is associated with poor survival and can be used as a prognostic factor in breast cancer. Medicine. 2015;94(11).

31. Wang C, Wang Y, Hong T, et al. Targeting a positive regulatory loop in the tumor-macrophage interaction impairs the progression of clear cell renal cell carcinoma. Cell Death Differ. 2020.

32. Morris MR, Ricketts C, Gentle D, et al. Identification of candidate tumour suppressor genes frequently methylated in renal cell carcinoma. Oncogene. 2010;29(14):2104-2117. doi:10.1038/onc.2009.493

33. Ricketts CJ, Hill VK, Linehan WM. Tumor-specific hypermethylation of epigenetic biomarkers, including SFRP1, predicts for poorer survival in patients from the TCGA Kidney Renal Clear Cell Carcinoma (KIRC) project. PLoS One. 2014;9(1):e85621. doi:10.1371/journal.pone.0085621
34. Urakami S, Shiina H, Enokida H, et al. Wnt antagonist family genes as biomarkers for diagnosis, staging, and prognosis of renal cell carcinoma using tumor and serum DNA. Clin Cancer Res. 2006;12 (23):6989-6997. doi:10.1158/1078-0432.CCR-06-1194

35. Hirata H, Hinoda Y, Nakajima K, et al. Wnt antagonist DKK1 acts as a tumor suppressor gene that induces apoptosis and inhibits proliferation in human renal cell carcinoma. Int J Cancer. 2011;128 (8):1793-1803. doi:10.1002/ijc.25507

36. Hoque MO, Begum S, Topaloglu O, et al. Quantitative detection of promoter hypermethylation of multiple genes in the tumor, urine, and serum DNA of patients with renal cancer. Cancer Res. 2004;64 (15):5511-5517. doi:10.1158/0008-5472.CAN-04-0799

37. Hauser S, Zahalka T, Fechner G, Muller SC, Ellinger J. Serum DNA hypermethylation in patients with kidney cancer: results of a prospective study. Anticancer Res. 2013;33(10):4651-4656.

38. Battagli C, Uzzo RG, Dulaimi E, et al. Promoter hypermethylation of tumor suppressor genes in urine from kidney cancer patients. Cancer Res. 2003;63(24):8695-8699.

\section{Publish your work in this journal}

OncoTargets and Therapy is an international, peer-reviewed, open access journal focusing on the pathological basis of all cancers, potential targets for therapy and treatment protocols employed to improve the management of cancer patients. The journal also focuses on the impact of management programs and new therapeutic agents and protocols on patient perspectives such as quality of life, adherence and satisfaction. The manuscript management system is completely online and includes a very quick and fair peer-review system, which is all easy to use. Visit http://www.dovepress.com/ testimonials.php to read real quotes from published authors. 